\title{
LAS REDES SOCIALES COMO FACTOR DE DECISIÓN: MILLENNIALS FRENTE A LA GENERACIÓN X
}

\author{
SOCIAL MEDIA LIKE DECISION FACTOR: MILLENNIALS \\ VERSUS X GENERATION.
}

Recibido: 15/08/2017

Aceptado: 22/11/2017
Pablo A. González Loyola ${ }^{1}$

(pablo.gonzalez@ucuenca.edu.ec)

Carlos A. Cañizares Alvarado²

(alberto.canizares92@gmail.com)

Gustavo A. Patiño Mosquera²

(gustavoandrespatino9@gmail.com)

\section{Resumen}

La importancia del desarrollo de las TIC ha marcado un cambio en los comportamientos de elección de compra de las personas. En distintos artículos, publicaciones y fuentes de información con enfoque social, de mercadotecnia, empresarial o cultural, hoy se habla de los Millennials, considerada la generación de mayor protagonismo en la sociedad actual. Sin embargo, antes que estos, se encuentran los conocidos como la Generación X.

Estos dos grupos generacionales, hoy, representan la mayor cantidad del mercado de consumo en la mayoría de sociedades. A través de una investigación concluyente, descriptiva y de corte trasversal simple que permite la comprobación de dos hipótesis planteadas, el presente trabajo demuestra que el principal factor por el que estas generaciones eligen lugares de interacción social como restaurantes, es diferente. Se descubre que una generación elige un restaurante por los comentarios en redes sociales y que otra generación lo hace por publicidad boca-oído que usan de forma distinta una misma red social y cómo el impacto de las redes sociales influye en su comportamiento social, comercial y de consumo.

Palabras Clave: Comportamiento, Consumo, Generación X, Influencia, Millennials, Redes sociales.

\begin{abstract}
The importance of ICT development has marked a change on consumer purchasing behavior. Different articles, publications and information sources, with a social, mercadotecnia, business or cultural focus, today analyses Millennials, which are considered the most important generation in today's society. However, before them there is the $X$ Generation. These two generational groups today represent the biggest quantity of market consumers in most societies. Through conclusive, descriptive and simple cross-section research, which allowed the affirmation of two hypotheses, the present document demonstrates that the main factor by which these generations choose places of social interaction such as restaurants is different. It is discovered that one generation chooses a restaurant because of social media comments and that the other one does it because of word of mouth, that they use the same social media in a different way and how its impact influences their social, commercial and consumption behavior.
\end{abstract}

Keywords: Behavior, Consumption, Influence, Millennials, Social Media, X Generation. 


\section{Introducción}

El vertiginoso desarrollo de la informática y de la tecnología en torno a la misma, principalmente en lo relacionado con las múltiples formas de comunicación que se han desarrollado a partir de la masificación del Internet y de las redes sociales, genera cambios en las sociedades de todo el planeta que, debido a la celeridad con la que se suscitan, en algunos casos, no han sido analizados a profundidad.

La presente investigación aborda un aspecto bastante coyuntural y limitado en cuanto al ámbito que abarca (Cuenca del Ecuador) y permitirá definir cuál es la incidencia de los comentarios en redes sociales, en la decisión de estratos poblacionales conformados por Millennials con estudios universitarios y personas de la Generación X, en la elección de restaurantes que aún no conocen.

El objetivo, en términos generales, es determinar hasta qué punto el fenómeno comunicacional que genera el uso de las diferentes redes sociales, puede tener incidencia en la decisión de una persona al elegir un restaurante que aún no conoce, según su generación.

Se considera el aspecto gastronómico porque, evidentemente, la alimentación y los gustos de las personas por determinadas variedades culinarias, son una parte esencial no solo de la cultura de una ciudad, sino también de su economía, ya que en torno a ella se proyectan y crean negocios que dinamizan la economía, porque mueven capitales y generan fuentes de empleo.

La selección de los segmentos poblacionales: Millennials con estudios universitarios y Generación X, se debe a que constituyen, un sector muy amplio de la población, específicamente representan juntos el 28 $\%$ de la población total del cantón Cuenca (142.575 personas aproximadamente) y son los que con mayor frecuencia concurren a restaurantes $\mathrm{y}$, como es obvio, se interesan por conocer la variedad o especialidad, así como su calidad culinaria.

Se, para el presente estudio, a la ciudad de Cuenca porque es una urbe con un estrato poblacional bastante profuso de clase media y tiene como valor agregado el hecho de ser una ciudad patrimonial con un fuerte atractivo para el turismo nacional e internacional, específicamente un $13,1 \%$ de los turistas internacionales visitó el Azuay, siendo un total de 204.416 visitantes (Guevara \& Morales, 2016); ello ha incidido positivamente en el incremento de emprendimientos de todo tipo, entre los que están los relacionados con el arte culinario y la gastronomía que tienen una significativa importancia en el desarrollo económico de la Ciudad.

\section{Revisión de literatura}

Existe una variedad de definiciones de redes sociales, todas ellas dependen del ángulo con el cual se las mire, sin embargo, entre los diferentes estudiosos del tema pueden encontrarse consensos, como por ejemplo el de calificarlas como sitios de Internet creados con la finalidad de dinamizar la comunicación y las relaciones entre los usuarios de la gran autopista virtual. Alberto Ureña, at/el (2011) refiriéndose a un artículo del Journal of Computer Mediated-Communication define a las redes sociales como:

Servicios dentro de las webs que permiten al usuario 1) construir un perfil público o semipúblico dentro de un sistema limitado, 2) articular una lista de otros usuarios con los que comparte una conexión y 3) visualizar y rastrear su lista de contactos y las elaboradas por otros usuarios dentro del sistema. (2011, pág. 12).

El objetivo central de las redes sociales es crear contactos entre la gente y crear grupos basándose en los más variados intereses que pueden ir desde el simple grupo de amigos hasta grupos con interés intelectual o de negocios.

Las redes no son únicamente un instrumento para entablar relaciones entre amigos o gente con intereses comunes, en realidad, "una red es una forma abstracta de visualizar una serie de sistemas, y en general, casi todos los sistemas complejos" (Merelo, 2006, p.1). En definitiva, las redes sociales han redefinido la forma en la que las personas interactúan entre sí, sin embargo, la esencia sigue siendo la misma: el 
intercambio de mensajes e información entre un grupo de personas; solo que ahora mediante la tecnología se han podido explorar nuevos medios, nuevas fuentes, nuevos recursos y nuevos canales.

Por otro lado, en cuanto a su clasificación, se puede decir que existen diferentes tipos de redes sociales. Según Burgueño (2009) una clasificación de las redes sociales de acuerdo con su objetivo y temática tendría dos ejes principales:

- Redes sociales horizontales: denomina así a aquellas redes sociales que van dirigidas a todo público y que además no tienen una temática específica, permitiendo participación libre y con el objetivo de generar masa. El ejemplo más claro de este tipo de red social es Facebook.

- Redes sociales verticales: en estas redes el objetivo principal es el de brindar un eje temático específico y bien definido, en el que los usuarios sean parte de esta red gracias a que están interesados en la temática de la red social. Un ejemplo de una red social vertical es Linkedln.

Con respecto a la publicidad, boca - oído, fue definida por primera vez como aquella comunicación oral de persona a persona entre un emisor que no se encuentra vinculado con la comercialización de un producto, servicio o marca y un receptor al que le interesa saber sobre dicho producto (Arndt, 1967). Este es un concepto de especial importancia para el presente estudio, ya que con el paso del tiempo ha evolucionado hasta el conocido como boca - oído electrónico que nace a merced de las nuevas vías de comunicación integradas desde el Internet y los avances tecnológicos. A la publicidad boca-oído electrónico se la debe entender como un comentario positivo o negativo realizado por personas ajenas a la empresa sobre una marca, producto, servicio u organización que puede estar disponible a una multitud de individuos e instituciones a través de Internet (Duan, Gu, \& Whinston, 2008).

Durante varios años se han realizado estudios para explicar por qué los consumidores utilizan la información que obtienen del boca - oído y del boca-oído electrónico. Como un hallazgo importante se destaca que tanto el boca-oído tradicional como el electrónico constituyen una fuente de información importante para los consumidores que puede, inclusive, resultar más influyente y confiable que la publicidad que utiliza medios de comunicación masiva o medios tradicionales (Goldsmith \& Horowitz, 2006).

Los estudios de publicidad boca-oído destacan la importancia del mismo en las decisiones de una persona que aún no ha consumido un servicio o un bien. Como lo explica Lau\&Ng (2001), los comentarios boca oído desempeñan un papel decisor en la aceptación de bienes o servicios. Las empresas no ignoran este fenómeno, por ello se han preocupado de utilizarlo en su favor.

Aguado y García (2009) definen el uso que se le ha dado al boca-oído tradicional y exponen que "ha sido utilizado como modo de publicidad efectiva sin recurrir a grandes inversiones ni contrataciones de medios masivos. Funciona al modo de una recomendación y gracias a esta, es posible adherir a nuestra cartera de clientes un cliente más" (Aguado Guadalupe \& García García, 2009, pág. 3).

Es de vital importancia resaltar el carácter bidimensional del boca-oído, pues se trata de una conducta con una dimensión evaluativa que se refiere al valor o grado de bondad que tiene la información, desde la más positiva a la más negativa, y por otro lado tiene una dimensión conativa que se puede denotar en el grado de difusión de la información a otros (Halstead, 2002). El boca-oído electrónico tendría un mayor grado de difusión, hablando de su dimensión conativa. Se deberá analizar tomando en cuenta el grado en el que la información está favoreciendo o perjudicando a la empresa y a cuántas personas les puede llegar esa información, como explican Aguado y García (2009) Internet ha modificado la práctica del boca - oído ampliando el alcance de la recomendación y permitiendo que se comparta en la dimensión de la web.

El boca-oído es una forma de comunicación que funciona solamente si el emisor quiere recomendar un producto y el receptor tiene alguna motivación para escuchar, es un acto que funciona en las dos direcciones (Villanueva \& Armelini, 2007). En el caso del boca-oído electrónico la iniciación desde el lado del receptor sería la búsqueda de información en las plataformas, puesto que, con los comentarios en redes sociales no se sabe a quién se está llegando, es decir, se quiere comentar con la satisfacción o insatisfacción en este caso del servicio de un restaurante, pero se emite la información a un público desconocido, anónimo; esta es la diferencia crucial con el boca - oído tradicional que implica una charla, "una recomendación 
hecha de amigo a amigo acerca de un producto implica confianza y credibilidad (...) basando su compra en la confianza depositada en la persona que referencia el mensaje." (Aguado Guadalupe \& García García, 2009, pág. 3), pero en el boca - oído electrónico los comentarios funcionarían como el registro de esa información, para que pueda ser consultada en el momento que se requiera, esporádicamente o por quién intencionalmente lo necesite, está abierta a todos los usuarios de la red.

Según Villanueva y Armelini, el receptor aceptará el consejo del emisor dependiendo de tres factores: la similitud existente entre ambos, el conocimiento del emisor sobre el producto en cuestión y el tipo de riesgo que percibe el receptor (Villanueva \& Armelini, 2007).

El riesgo percibido se puede clasificar en varios tipos, pero a efectos de la investigación se nombrará a los dos más comunes: riesgo financiero y riesgo social. El riesgo financiero es "la posibilidad de que el producto no valga lo que cuesta" (Leon \& Leslie, 2010, págs. 183-184) y el riesgo social es el "peligro de que una decisión errónea sobre productos genere una situación socialmente embarazosa" (Leon \& Leslie, 2010, págs. 183-184). Dependiendo del tipo de riesgo que el consumidor perciba, se determinará la fuente de información a la que se acuda. Cuando una persona perciba un riesgo social, será más probable que busque consejo de algún conocido, familiar o persona cercana, es decir, publicidad boca - oído ya sea tradicional o electrónico; por otro lado, si es que el individuo percibe un riesgo financiero, buscará consejo de personas expertas o fuentes especializadas (Villanueva \& Armelini, 2007).

La manera en que una persona intenta reducir su riesgo percibido funciona de igual manera en el bocaoído electrónico y en el boca-oído tradicional. Cuando se presenta un riesgo social percibido, un individuo puede minimizar dicho riesgo interactuando con personas que comparten intereses en común y las pueden encontrar de manera más fácil y rápida en plataformas electrónicas como las redes sociales (Villanueva \& Armelini, 2007).

Moliner-Velázquez (2012) realizó un estudio titulado "El boca-oído de clientes insatisfechos: un enfoque de segmentación en servicios de restaurantes", en donde afirma que el boca - oído es causa y consecuencia de la experiencia de los consumidores. Es causa cuando perciben riesgo, por lo que antes de comprar, los consumidores, para reducir el riesgo, consultan información de otros. Es consecuencia después de comprar y tiene distintos fines: para brindar ayuda a otros consumidores, vengarse de la empresa, desahogarse respecto al servicio o para reducir la inseguridad de compradores. Además, señala que los compradores insatisfechos tienden más a comentar sus experiencias que los compradores satisfechos, por lo que el boca - oído negativo es más frecuente y tiene mayor efecto en los consumidores que el boca - oído positivo.

En términos más generales el consumidor actual ya no es un consumidor pasivo. Hoy busca, investiga y acopia su propia información usando las TICs. El consumidor pasivo ha muerto desde que se dio cuenta que no necesita ni de intermediarios, ni de publicidad para conseguir su producto (Rattinger, 2013).

Esta forma en la que los consumidores responden ante los servicios de las empresas y los nuevos medios que brinda la tecnología, ha aportado para que se construya el escenario en el que se da el fenómeno conocido como "prosumidores", como lo afirma el consultor internacional experto en nuevo marketing, Juan Sánchez, en su artículo "Comprender el Marketing de Boca a Oído", en donde sostiene que los consumidores han evolucionado y se han convertido en productores de información, consecuencia del desarrollo de herramientas de publicación de contenido, como por ejemplo los blogs, con audiencias que en algunos casos tienen un gran alcance y con poderes de influencia que permiten viralizar información con mayor poder de credibilidad que cualquier comunicación publicitaria de las empresas y posicionar recomendaciones como el arma del mercadotecnia más efectiva (Sánchez, 2009).

La palabra "prosumidor" es un acrónimo de "productor" y "consumidor", el término fue concebido en primera instancia en inglés "prosumer" resultado de "produce" $\mathrm{y}$ "consumer", por el futurólogo Alvin Toffler en su libro "La Tercera Ola", publicado en 1981.

En el libro se expone que durante la primera ola las personas eran consumidoras de su propia producción hasta que sobrevino la revolución industrial y junto con ella se separaron los papeles de lo que ahora conocemos como productores y consumidores, en donde además claramente eran los productores los 
que, a través de los medios disponibles, emitían mensajes para los consumidores. Durante la segunda ola la televisión era el medio de comunicación más poderoso y masificador de la sociedad. Durante la tercera ola es en donde los "prosumidores", a partir de los medios de comunicación generados, empiezan a destruir la hegemonía de la producción de mensajes de los medios de comunicación de la segunda ola (Islas, 2008).

Finalmente, en lo que respecta a los términos Generación X y Millennials, estos son utilizados para referirse a las cohortes demográficas que incluyen a los nacidos entre 1965 y 1981 y a los nacidos entre 1982 y 1998, respectivamente, según Antoni Gutiérrez-Rubí (2016). Esto quiere decir que para el año 2017, los Millennials tienen entre 19 y 35 años, mientras que las personas de la Generación X tienen entre 36 y 52 años.

En términos estrictamente académicos, la denominación "Millennials" aparece por primera vez en el libro de los demógrafos Neil Howe y William Strauss "Millennials Rising: The Next Great Generation", publicado en el año 2000, a quienes se les adjudica la invención de este concepto; la razón por la que se los calificó así fue porque es la generación que vivió el cambio de milenio.

Existen características que se repiten en esta generación que permiten generar una persona ideal que representa a este cohorte demográfico y que permite comprender de mejor manera su comportamiento, recalcando que "por supuesto que habrá excepciones, muchas, así como también puntos en común con otras generaciones, pero vale la pena hacer el esfuerzo y asumir los riesgos" (Gutiérrez, 2016, pág. 5).

\section{Materiales y Métodos}

La metodología propuesta, inicia con el planteamiento de dos hipótesis, las mismas serán puestas a prueba mediante las herramientas estadísticas: prueba de hipótesis para dos muestras independientes y chi-cuadrado de Pearson; además, para el análisis de los datos será conveniente el uso del software estadístico SPSS versión 21.

Es importante mencionar la elección del método de muestreo, que después de varios análisis se establece y es la técnica de muestreo por estratos. El empleo de todas estas herramientas se especifica en anexos con la ficha técnica de la investigación.

Considerando toda la información acerca del distinto comportamiento de ambas generaciones y tomando en cuenta la penetración de la tecnología, se plantea la inquietud acerca de cómo ha influido esto en el comportamiento de la comunidad local. Es decir, qué tanto influyen los comentarios en redes sociales o boca - oído electrónico y el boca - oído tradicional en los Millennials y la Generación X de la ciudad de Cuenca. Es posible que exista una diferencia en la credibilidad que se otorga a ambos fenómenos y que esta credibilidad esté en función de la generación a la que se pertenece, por lo que se determinan las siguientes hipótesis:

H1: Los comentarios en redes sociales son el principal factor en la toma de decisiones de los Millennials.

H2: El boca - oído tradicional es el principal factor en la toma de decisiones de la Generación X.

Para probar estas hipótesis se ha decidido desarrollar una investigación concluyente, descriptiva y de corte transversal simple. Además, se contará con un proceso de investigación formal y estructurada con análisis de datos cuantitativos con lo que se obtendrán hallazgos concluyentes útiles para tomar decisiones.

Para determinar el universo que será de utilidad al calcular el tamaño de la muestra, se parte de la población total del cantón Cuenca que es de 505.585 personas, de ese número, se incluye al número de personas que corresponden a los dos grupos etarios de interés que juntos representan aproximadamente el $28 \%$ de la población total, por lo que el universo será de 142.575 personas, por otro lado, la población de estudio está determinada por personas de los dos grupos de interés, Millennials y Generación X:

- Millennials: Personas que viven en Cuenca - Ecuador, dentro del casco urbano, de ambos géneros, con edades comprendidas entre 25 y 35 años, con estudios universitarios (65\% del universo). 
- Generación X: Personas que viven en Cuenca - Ecuador, dentro del casco urbano, de ambos géneros, con edades comprendidas entre 40 y 50 años (35\% del universo).

Con estos datos se ha utilizado el método de muestreo estratificado para el cálculo de la muestra, con un nivel de significancia de $95 \%$ y un error máximo admisible de $5 \%$. Además, para determinar los valores de la probabilidad de éxito y de fracaso ( $\mathrm{p}$ y q), se ha tenido en cuenta una prueba piloto realizada con anterioridad, en la que se determinó que la probabilidad de éxito en el caso de los Millennials, es de $70 \%$, mientras que, en el caso de la Generación X, es del $80 \%$.

La probabilidad de éxito en el caso de los Millennials está determinada por el porcentaje de personas que consideran a los comentarios en redes sociales como principal factor para escoger un restaurante que aún no conocen. Para la Generación X, la probabilidad de éxito será el porcentaje de personas que consideran a la publicidad boca - oído el principal factor para escoger un restaurante que aún no conocen. El resultado obtenido, da una muestra de 295 personas, correspondiente a la siguiente fórmula:

$$
n=\frac{\sum\left(w_{i} * p_{i} * q_{i}\right)}{\frac{e^{2}}{z^{2}}+\frac{\sum\left(w_{i} * p_{i} * q_{i}\right)}{N}}
$$

Reemplazando los datos:

$$
n=\frac{(0,65 * 0,70 * 0,30)+(0,35 * 0,80 * 0,20)}{\frac{0,05^{2}}{1,96^{2}}+\frac{(0,65 * 0,70 * 0,30)+(0,35 * 0,80 * 0,20)}{142575}}=295
$$

Finalmente, para la comprobación de las dos hipótesis planteadas en el estudio, se usa principalmente dos herramientas estadísticas: prueba de hipótesis para muestras independientes y prueba de independencia chi-cuadrado. El detalle del uso que se dará dependerá de las variables que se vayan a medir, las mismas se explicarán más adelante.

Para la determinación de las características de los dos grupos de estudio se utilizan las mismas herramientas, además de estadísticos descriptivos. Las variables que se utilizaron para esto serán detalladas más adelante.

\subsection{Prueba de hipótesis para dos muestras independientes}

Para comprobar si existen diferencias entre las variables según los grupos de estudio, se utiliza la herramienta estadística "prueba de hipótesis para dos medias con muestras independientes", cuya función radica en obtener las medias y desviaciones estándar de cada uno de los grupos de estudio y las compara entre sí para determinar si es que existen diferencias estadísticamente significativas (Webster, 2000). Las hipótesis que se plantean para esta prueba son las siguientes:

$$
H_{0} ; \mu_{\text {Millennials }}=\mu_{(\text {Generación } X)}
$$

Ho: No existe diferencias significativas entre las medias de los Millennials y las medias de la Generación X.

$$
H_{a} ; \mu_{\text {Millennials }} \neq \mu_{(\text {Generación X) }}
$$

Ha: Existe diferencias significativas entre las medias de los Millennials y las medias de la Generación X.

Donde " $\mu$ " es la media de las calificaciones.

Para determinar si existen diferencias significativas entre las medias de las calificaciones del grupo de los Millennials frente al grupo de la Generación X, será necesario calcular el valor $t$ de cada una de las variables a probar (las variables ver en tabla 1 y tabla 2) y contrastar con el valor $Z$ de tabla. El valor de 
$Z$ de tabla para un nivel de confianza del $95 \%$ es de \pm 1.96 , mientras que el valor $t$ es calculado con la comparación de las medias y desviaciones estándar de cada variable que se va a probar.

De esta manera la regla de decisión es que si el valor t calculado es menor que -1.96 y mayor que +1.96 la hipótesis nula se rechaza, por lo tanto, se acepta que existen diferencias significativas entre las medias de los dos grupos. Por otro lado, si es que el valor t calculado está dentro del rango de \pm 1.96 no se rechaza la hipótesis nula y se concluiría que no existen diferencias significativas entre las medias de los dos grupos de estudio.

Cabe recalcar que las variables a medir con esta herramienta fueron hechas con una escala de Likert donde: 1 = muy de acuerdo, $2=$ de acuerdo, $3=$ ni de acuerdo ni en desacuerdo, $4=$ en desacuerdo y $5=$ muy en desacuerdo.

\subsection{Chi-cuadrado de Pearson}

La distribución chi-cuadrado es una herramienta no paramétrica ${ }^{3}$ que en este estudio será útil para comparar dos variables cualitativas y determinar si es que existe independencia entre ellas. Las variables que se van a medir con esta herramienta estadística serán explicadas más adelante (ver tabla 1 y tabla 2).

Para esto es necesario plantearse las hipótesis de la herramienta de la siguiente manera:

Ho: La generación a la que pertenecen y la variable de contraste son independientes.

Ha: La generación a la que pertenecen y la variable de contraste no son independientes.

Para probar las hipótesis se calculará un valor de chi-cuadrado junto con un valor de significancia asintótica bilateral (valor p), con un error máximo admisible del $5 \%=0,05$ que forma la siguiente regla de decisión:

No rechazar la hipótesis nula, si es que el valor de la significancia asintótica bilateral es mayor que 0,05.

Rechazar la hipótesis nula, si es que el valor de la significancia asintótica bilateral es menor que 0,05.

Finalmente, para la resolución de la primera hipótesis planteada en el estudio, se presenta la siguiente metodología:

$\mathrm{H} 1$ : Los comentarios en las redes sociales son el principal factor por el que el $70 \%$ de los Millennials escogen un restaurante que aún no conocen.

Para comprobar esta hipótesis se plantean los siguientes indicadores con sus respectivas preguntas presentes en la encuesta usada.

Tabla 1. Indicadores, preguntas y herramienta estadística para la comprobación de la primera hipótesis

\begin{tabular}{|l|c|c|}
\hline \multirow{2}{*}{ Indicadores } & $\begin{array}{c}\text { Preguntas de la } \\
\text { encuesta }\end{array}$ & Herramienta estadística a utilizar \\
\hline \multirow{2}{*}{ Uso de redes sociales } & 7 & Prueba de independencia Chi-cuadrado \\
\cline { 2 - 3 } & 2 & Prueba de independencia Chi-cuadrado \\
\hline \multirow{2}{*}{ Fuentes de información } & 9 & Prueba de hipótesis para dos muestras independientes \\
\cline { 2 - 3 } & $14 a$ &
\end{tabular}

3 Prueba no paramétrica: Es un procedimiento estadístico útil para probar hipótesis cuando los supuestos de parámetros o distribuciones poblacionales E ñoson posibles (Wébstêr, 2000)No. 27 


\begin{tabular}{|c|c|l|}
\hline $\begin{array}{c}\text { Importancia de las redes sociales } \\
\text { y la publicidad boca-Oído. }\end{array}$ & 15a. & Prueba de hipótesis para dos muestras independientes \\
\hline $\begin{array}{c}\text { Confianza en la publicidad Boca- } \\
\text { Oído y comentarios en redes } \\
\text { sociales. }\end{array}$ & $15 \mathrm{~b}$ & Prueba de hipótesis para dos muestras independientes \\
\hline $\begin{array}{c}\text { Comodidad de consulta de } \\
\text { comentarios en Redes Sociales } \\
\text { frente a la publicidad Boca-Oído }\end{array}$ & $15 \mathrm{~d}$ & Prueba de hipótesis para dos muestras independiententes \\
\hline $\begin{array}{c}\text { Nivel de influencia de comentarios } \\
\text { en redes sociales. }\end{array}$ & $16 \mathrm{a}$ & Prueba de independencia Chi-cuadrado \\
\hline $\begin{array}{c}\text { Factor principal por el que se } \\
\text { escoge un restaurante que no } \\
\text { conoce. }\end{array}$ & 17 & \\
\hline
\end{tabular}

Elaboración: Los autores

La tabla 1 explica el camino a seguir para la comprobación de la primera hipótesis planteada en el estudio que consta de 9 preguntas.

Para la resolución de la segunda hipótesis planteada en el estudio, se presenta la siguiente metodología: $\mathrm{H}_{2}$ : La publicidad boca - oído es el principal factor por el que el $80 \%$ de las personas de la Generación X escogen un restaurante que aún no conocen.

Para comprobar esta hipótesis se plantean los siguientes indicadores con sus respectivas preguntas presentes en la encuesta usada para el efecto.

Tabla 2: Indicadores, preguntas y herramienta estadística para la comprobación de la segunda hipótesis

\begin{tabular}{|c|c|c|}
\hline Indicadores & Preguntas de la encuesta & Herramienta estadística a utilizar \\
\hline Fuentes de información & 9 & Prueba de independencia Chi-cuadrado \\
\hline $\begin{array}{c}\text { Importancia de las redes } \\
\text { sociales y la publicidad boca } \\
\text { oído. }\end{array}$ & $15 \mathrm{a}$. & Prueba de hipótesis para dos muestras independientes \\
\hline $\begin{array}{c}\text { Confianza en la publicidad } \\
\text { Boca oído y comentarios en } \\
\text { redes sociales. }\end{array}$ & $5 \mathrm{~b}$ & Prueba de hipótesis para dos muestras independientes \\
\cline { 2 - 3 } & $15 \mathrm{c}$ & Prueba de hipótesis para dos muestras independientes \\
\hline $\begin{array}{c}\text { Comodidad de comentarios } \\
\text { en Redes sociales frente a la } \\
\text { publicidad boca oído. }\end{array}$ & $15 \mathrm{~d}$. & Prueba de hipótesis para dos muestras independientes \\
\hline $\begin{array}{c}\text { Nivel de influencia de } \\
\text { comentarios en redes sociales } \\
\text { y boca oído. }\end{array}$ & $16 \mathrm{a}$ & Prueba de hipótesis para dos muestras independientes \\
\cline { 2 - 4 }
\end{tabular}


Factor principal por el que se escoge un restaurante que no conoce.
Prueba de independencia Chi-cuadrado

Elaboración: Los autores

La tabla 2 explica el camino a seguir para la comprobación de la segunda hipótesis planteada en el estudio que consta de 8 preguntas.

\section{Discusión y resultados}

4.1 Primera hipótesis: Comentarios en redes sociales como principal factor de decisión para los Millennials. La hipótesis fue planteada de la siguiente manera:

Ho: Los comentarios en las redes sociales no son el principal factor por el que el $70 \%$ de los Millennials escogen un restaurante que aún no conocen.

H1: Los comentarios en las redes sociales son el principal factor por el que el $70 \%$ de los Millennials escogen un restaurante que aún no conocen.

Como se explicó en la sección anterior, se han realizado pruebas de hipótesis y pruebas de independencia chi-cuadrado para las variables y preguntas útiles para resolver esta hipótesis. En las primeras preguntas a analizar, se encontró que, según la prueba de independencia chi-cuadrado, existe relación entre la variable "generación a la que pertenece" con las variables: "uso de redes sociales", "fuentes de información en las que confía" y "revisó comentarios en su última visita a un restaurante" (Ver tabla 3).

Analizando esto se sabe que la mayoría de Millennials usa redes sociales para revisar información de locales comerciales (horarios, dirección, servicios) y para chatear, además al momento de buscar información acerca de un restaurante que aún no conocen, consideran confiables a los comentarios en redes sociales y recomendaciones de terceros. Finalmente se sabe que la última vez que visitaron un restaurante revisaron sus comentarios en redes sociales.

Tabla 3: Prueba de independencia chi-cuadrado

\begin{tabular}{|c|c|c|c|}
\hline Variables & Millennials & Sig. Asintótica & GI. \\
\hline $\begin{array}{c}\text { Uso que le dan a las redes } \\
\text { sociales* }\end{array}$ & $\begin{array}{c}\text { Revisar información de locales } \\
\text { comerciales (horarios, dirección, } \\
\text { servicios). }\end{array}$ & 0,000 & 11 \\
\hline $\begin{array}{c}\text { Fuentes de información } \\
\text { confiable* }\end{array}$ & Comentarios en redes sociales. & 0,000 & 5 \\
\hline $\begin{array}{c}\text { La última vez que visitó } \\
\text { un restaurante, revisó los } \\
\text { comentarios en redes sociales* }\end{array}$ & Sí & 0,000 & 1 \\
\hline
\end{tabular}

${ }^{*}$ Chi-cuadrado significativo al $99 \%$

Elaboración: Los autores

Para el análisis de las siguientes variables, se utilizó la herramienta prueba de hipótesis para dos muestras independientes, encontrando diferencias estadísticamente significativas en sus respuestas (Ver tabla 4), gracias a esto se sabe que: 
Los Millennials están de acuerdo en lo siguiente: las redes sociales son buena fuente de información acerca de restaurantes que aún no conocen, es importante leer los comentarios en redes sociales acerca del servicio de un restaurante que aún no conocen, confían en los comentarios en redes sociales respecto a un restaurante que aún no conocen.

Además, no están ni de acuerdo ni en desacuerdo en que es más cómodo revisar los comentarios en redes sociales en lugar de preguntar a un desconocido acerca de un restaurante que aún no conocen y están de acuerdo en que los comentarios en redes sociales de otros usuarios influyen en su decisión de acudir a un restaurante que aún no conocen.

Tabla 4: Valores t calculados

\begin{tabular}{|c|c|c|}
\hline Variables & Millennials & Valor t \\
\hline $\begin{array}{c}\text { Las redes sociales de restaurantes son buena fuente de } \\
\text { información }\end{array}$ & 1,72 & $*-6,811$ \\
\hline $\begin{array}{c}\text { Es importante leer los comentarios en redes sociales acerca del } \\
\text { servicio de un restaurante que aún no conoce }\end{array}$ & 2,01 & $*-4,817$ \\
\hline $\begin{array}{c}\text { Confío en los comentarios en redes sociales respecto a un } \\
\text { restaurante que aún no conozco }\end{array}$ & 2,46 & $*-5,211$ \\
\hline $\begin{array}{c}\text { Al momento de acudir a un restaurante que aún no conozco, es } \\
\text { más cómodo revisar los comentarios en las redes sociales del } \\
\text { restaurante, que preguntar a algún conocido. }\end{array}$ & 2,57 & $*-2,408$ \\
\hline $\begin{array}{c}\text { Los comentarios y opiniones de otros usuarios en las redes sociales } \\
\text { influyen en mi decisión de acudir a un restaurante que aún no } \\
\text { conozco }\end{array}$ & 2,42 & $*-7,161$ \\
\hline
\end{tabular}

\section{* Z crítico a $\pm 1,96$}

\section{Elaboración: Los autores}

Finalmente, realizando un análisis chi-cuadrado entre la variable "generación a la que pertenece" y la variable "factor principal al momento de escoger un restaurante que aún no conoce", se determina que hay una relación estadísticamente significativa y que para el $47,4 \%$ de los Millennials el principal factor son los comentarios en redes sociales.

Tabla 5: Prueba de independencia chi-cuadrado

\begin{tabular}{|c|c|c|c|}
\hline Variables & Millennials & $\begin{array}{c}\text { Sig. } \\
\text { Asintótica }\end{array}$ & Gl \\
\hline $\begin{array}{c}\text { Factor principal al momento de escoger un } \\
\text { restaurante que aún no conocen* }\end{array}$ & $\begin{array}{c}\text { Comentarios en redes } \\
\text { sociales }\end{array}$ & 0,000 & 3 \\
\hline
\end{tabular}

${ }^{*}$ Chi-cuadrado significativo al $99 \%$

Elaboración: Los autores 
4.2 Segunda hipótesis: Publicidad boca - oído como principal factor de decisión para la Generación X.

La hipótesis fue planteada de la siguiente manera:

H0: La publicidad boca-oído no es el principal factor por el que el $80 \%$ de las personas de la Generación $X$ escogen un restaurante que aún no conocen.

H1: La publicidad boca-oído es el principal factor por el que el $80 \%$ de las personas de la Generación X escogen un restaurante que aún no conocen.

Al igual que en la primera hipótesis, para resolver esta se han utilizado las herramientas estadísticas: pruebas de hipótesis y pruebas de independencia chi-cuadrado. En la primera variable se realiza una prueba de independencia chi-cuadrado y se determina que existe relación entre la variable "generación a la que pertenece" y la variable "fuentes de información en las que confía" ya que las personas de la Generación X confían en las recomendaciones de terceros (Publicidad boca-oído).

Tabla 6: Prueba de independencia chi-cuadrado

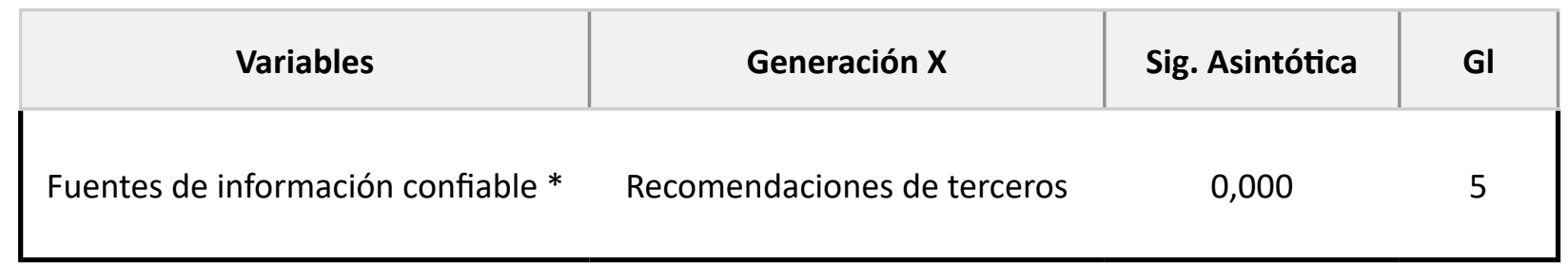

* Chi-cuadrado significativo al $99 \%$

Elaboración: Los autores

Para el análisis de las siguientes variables, se utilizó la herramienta estadística prueba de hipótesis para dos muestras independientes, encontrando diferencias estadísticamente significativas en sus respuestas (Ver tabla 7), gracias a esto se sabe que:

Las personas de la Generación X no están ni de acuerdo ni en desacuerdo con dos factores: 1) es importante revisar los comentarios en redes sociales acerca del servicio de un restaurante que aún no conocen y 2) confían en los comentarios en redes sociales respecto a un restaurante que aún no conocen (Ver tabla 7).

Además, no están de acuerdo ni en desacuerdo con el hecho de que revisar los comentarios en redes sociales es más cómodo que preguntar a un conocido acerca de un restaurante que aún no conoce. También en que los comentarios en redes sociales de otras personas influyen en la decisión de acudir a un restaurante que aún no conocen (Ver tabla 7).

Por otro lado, no se encontraron diferencias estadísticamente significativas en estas dos variables: 1) confían en las recomendaciones de terceros respecto a un restaurante que aún no conocen y 2) las recomendaciones de terceras personas influyen en su decisión de acudir a un restaurante que aún no conocen, estando de acuerdo con esas dos afirmaciones (Ver tabla 7). 
Tabla 7: Valores t calculados

\begin{tabular}{|c|c|c|}
\hline & Generación X & Valor t \\
\hline $\begin{array}{c}\text { Es importante leer los comentarios en redes sociales acerca del } \\
\text { servicio de un restaurante que aún no conoce }\end{array}$ & 2,58 & * $-4,817$ \\
\hline $\begin{array}{l}\text { Confío en los comentarios en redes sociales respecto a un restaurante } \\
\qquad \text { que aún no conozco }\end{array}$ & 3,12 & * $-5,211$ \\
\hline $\begin{array}{l}\text { Confío en las recomendaciones de terceros respecto a un restaurante } \\
\text { que aún no conozco }\end{array}$ & 2,21 & -1.853 \\
\hline $\begin{array}{c}\text { Al momento de acudir a un restaurante que aún no conozco, es más } \\
\text { cómodo revisar los comentarios en las redes sociales del restaurante, } \\
\text { que preguntar a algún conocido. }\end{array}$ & 2,88 & $*-2,408$ \\
\hline $\begin{array}{l}\text { Los comentarios y opiniones de otros usuarios en las redes sociales } \\
\text { influyen en mi decisión de acudir a un restaurante que aún no conozco }\end{array}$ & 3,56 & $*-7,161$ \\
\hline $\begin{array}{l}\text { Las recomendaciones de terceras personas influyen en mi decisión de } \\
\text { acudir a un restaurante que aún no conozco }\end{array}$ & 2,3 & -1.165 \\
\hline
\end{tabular}

* $Z$ crítico $\pm 1,96$

Elaboración: Los autores

Finalmente, realizando un análisis chi-cuadrado entre la variable "generación a la que pertenece" y la variable "factor principal al momento de escoger un restaurante que aún no conoce", se tiene que hay una relación estadísticamente significativa y que para el 92,20\% de las personas de la Generación X el principal factor es la publicidad boca - oído.

Tabla 8: Prueba de independencia chi-cuadrado

\begin{tabular}{|c|c|c|c|}
\hline Variables & Generación X & Sig. Asintótica & Gl \\
\hline $\begin{array}{c}\text { Factor principal al momento de escoger un } \\
\text { restaurante que aún no conocen*. }\end{array}$ & $\begin{array}{c}\text { Recomendaciones de } \\
\text { terceros }\end{array}$ & 0,000 & 3 \\
\hline
\end{tabular}

* Chi-cuadrado significativo al $99 \%$

Elaboración: Los autores 


\subsection{Características de los Millennials y la Generación X.}

En esta sección se detallan características generales obtenidas en la investigación de mercados y que serán de utilidad para tener una aproximación al perfil de gustos y preferencias de los dos grupos de estudio respecto a las redes sociales.

En las variables de la tabla 9 se realizó la prueba de independencia chi-cuadrado, existiendo relación estadísticamente significativa entre la variable "generación a la que pertenecen" y las variables: "dispositivo con el que prefiere navegar en redes sociales", "redes sociales preferidas" y "sigue las redes sociales de restaurantes locales". Con esos datos se obtienen los siguientes resultados:

Tanto Millennials como Generación X prefieren el celular para navegar en sus redes sociales, las redes sociales de preferencia para los Millennials, en orden de importancia, son: Facebook, YouTube e Instagram, mientras que para la Generación X, en orden de importancia, son: Facebook, YouTube y Google+.

En promedio, los Millennials usan 4 redes sociales, mientras que, los de la Generación X usan 3. La red social más utilizada por los dos grupos es Facebook, usan de 1 a 2 horas al día y el horario preferido es en las noches. El uso preferido que dan a las redes sociales es para chatear.

Por otro lado, los Millennials aseguran seguir a redes sociales de restaurantes locales, mientras que la Generación X no lo hace. Además, la red social preferida por los dos grupos para revisar información acerca de restaurantes locales que no conocen es Facebook.

Tabla 9: Descriptivo de los dos grupos

\begin{tabular}{|c|c|c|}
\hline $\begin{array}{c}\text { Dispositivo con el que prefiere navegar en redes } \\
\text { sociales }\end{array}$ & Millennials & Generación X \\
\hline Redes sociales preferidas* & 1. Facebook & 1. Facebook \\
\hline Promedio de redes sociales que usan & 2. YouTube & 3. Google+ \\
\hline Red social más utilizada & 3. Instagram & 4. Twitter \\
\hline Tiempo al día que usan redes sociales & Facebook & \\
\hline Horario preferido para visitar redes sociales & En las noches (18ho0 a 00h00) & \\
\hline Uso más frecuente que dan a sus redes sociales & De 1 a 2 horas & \\
\hline Sigue a redes sociales de algún restaurante local* & Chatear & \\
\hline restaurantes que no conocen & & \\
\hline
\end{tabular}

* Chi-cuadrado significativo al $99 \%$

Elaboración: Los autores. 
En las variables de la tabla 10 se utilizaron "pruebas de hipótesis", existiendo diferencias estadísticamente significativas entre la media de la variables "generación a la que pertenecen" con las variables: "utilizo mucho redes sociales para seguir a restaurantes locales", "los restaurantes locales con redes sociales inspiran más confianza", "la publicidad en redes sociales es muy molesta", "la publicidad en redes sociales es útil para conocer nuevos productos", "las redes sociales de los restaurantes son un buen lugar para opinar acerca de su servicio" y "confío en los comentarios en las redes sociales de restaurantes, aún si el comentario viene de alguien que no conozco". Con esos datos se obtuvieron los siguientes resultados.

Los Millennials están de acuerdo en que utilizan mucho las redes sociales para seguir a restaurantes locales, mientras que la Generación X está en desacuerdo con esta afirmación. De igual manera, los Millennials están de acuerdo en que los restaurantes locales con redes sociales inspiran más confianza que aquellos que no tienen redes sociales, mientras que la Generación X no está de acuerdo ni en desacuerdo con esto.

En cuanto al hecho de que la publicidad en redes sociales es muy molesta, los Millennials afirman estar de acuerdo, mientras que la Generación X no está de acuerdo ni en desacuerdo, sin embargo, la publicidad en redes sociales es útil para conocer nuevos productos, es algo que los Millennials están "muy de acuerdo" y la Generación X "de acuerdo".

Los Millennials están de acuerdo en que las redes sociales de los restaurantes son un buen lugar para opinar acerca de su servicio, mientras que para la Generación X esto le es indiferente. Además, los dos grupos de estudio están de acuerdo en que confía más en recomendaciones en persona de conocidos que de desconocidos.

Finalmente, en cuanto a la confianza de los comentarios en redes sociales de restaurantes, los Millennials están de acuerdo en que confían así el comentario venga de alguien que no conocen, mientras que la Generación X está en desacuerdo con esta afirmación.

Tabla 10: Valores t calculados

\begin{tabular}{|c|c|c|c|}
\hline Utilizo mucho redes sociales para seguir a restaurantes locales & 2,43 & 3,72 & $*-8,911$ \\
\hline $\begin{array}{c}\text { Los restaurantes locales con redes sociales inspiran más } \\
\text { confianza. }\end{array}$ & 2,34 & 2,98 & $*-5,086$ \\
\hline $\begin{array}{c}\text { La publicidad en redes sociales es muy molesta. } \\
\text { La publicidad en redes sociales es útil para conocer nuevos } \\
\text { productos. }\end{array}$ & 2,42 & 3,09 & $*-2,103$ \\
\hline $\begin{array}{c}\text { Las redes sociales de los restaurantes son un buen lugar para } \\
\text { opinar acerca de su servicio }\end{array}$ & 2 & 2,06 & $*-2,725$ \\
\hline $\begin{array}{c}\text { Si la recomendación acerca de un restaurante es en persona. } \\
\text { Confío más en un conocido que en un desconocido. }\end{array}$ & 1,8 & 1,66 & $*-4,428$ \\
\hline $\begin{array}{c}\text { Confío en los comentarios en las redes sociales de restaurantes, } \\
\text { aún si el comentario viene de alguien que no conozco. }\end{array}$ & 2,37 & 3,75 & $*-5,054$ \\
\hline
\end{tabular}

Z crítico $\pm 1,96$

Elaboración: Los autores. 


\section{Conclusiones y recomendaciones}

\subsection{Comentarios en redes sociales como factor de decisión de los Millennials}

La primera hipótesis pretendía comprobar si es que los comentarios en redes sociales son el principal factor por el que el $70 \%$ de los Millennials escogen un restaurante que aún no conocen, siguiendo la metodología presentada anteriormente se tienen las siguientes conclusiones.

Las redes sociales de los Millennials están presentes antes de sus decisiones. El uso que los Millennials dan a las redes sociales es para revisar información de locales comerciales, es decir, sus horarios, dirección y/o servicios. Además, la última vez que visitaron un restaurante aproximadamente el 58\% revisó los comentarios en redes sociales del mismo antes de acudir.

Los comentarios en redes sociales son parte de la búsqueda de información durante el proceso de compra de los Millennials. Las fuentes que les parecen confiables al momento de buscar información acerca del servicio de un restaurante que no conocen son principalmente los comentarios en redes sociales con un 61 $\%$, sin embargo, la mitad de los Millennials también consideran que las recomendaciones de terceros son una fuente confiable. Además, están de acuerdo en que las redes sociales de los restaurantes son buena fuente de información, esto tiene coherencia con el uso que le dan a las redes, expuesto anteriormente.

A pesar de que hay una clara tendencia hacia revisar los comentarios en redes sociales antes de visitar un restaurante, aún es mejor preguntar a terceras personas. Los Millennials están de acuerdo en que es importante leer los comentarios en las redes sociales acerca de un restaurante que aún no conocen, además están de acuerdo en que confían en los mismos, esto concuerda también con el uso que le dan y la confianza que tienen con las redes sociales. Sin embargo, no les parece más cómodo revisar los comentarios en redes sociales que preguntar a algún conocido.

Los comentarios en redes sociales resultan influyentes e importantes para los Millennials. Están de acuerdo en que los comentarios y opiniones de otros usuarios influyen en su decisión de acudir a un restaurante que aún no conocen. Además, el factor principal para escoger un restaurante que aún no conocen para los Millennials está dividido: una parte mayoritaria asegura que son los comentarios en redes sociales $(47,4 \%)$, mientras que, otro grupo importante dice que el principal factor es la publicidad boca - oído $(41,10 \%)$.

En base a los datos expuestos, la hipótesis de que los comentarios en las redes sociales son el principal factor por el que el $70 \%$ de los Millennials escogen un restaurante que aún no conocen, se tiene que rechazar, ya que no les parece más cómodo revisar los comentarios en redes sociales y tan sólo el $47,4 \%$ lo considera como su principal factor.

A pesar de esto, es claro que existe una tendencia favorable hacia este uso de las redes sociales, sin embargo, con los datos analizados parece ser que también es importante los comentarios de terceros (o publicidad boca - oído). Es posible que los Millennials en Cuenca estén en una transición desde la publicidad boca - oído, hacia la publicidad boca - oído electrónica.

En base a las conclusiones antes citadas, se recomienda motivar a los clientes a que sigan las redes sociales del restaurante y a que califiquen o realicen comentarios acerca de los servicios según su satisfacción, lo que les llamó la atención, lo que más le gustó del servicio, etc., para esto es necesario establecer un sistema de recompensas basado en entregar beneficios valorados por sus clientes a cambio de dichas calificaciones y comentarios.

\subsection{La publicidad boca-oído como factor de decisión de la Generación X}

La segunda hipótesis pretendía comprobar si es que la publicidad boca-oído es el principal factor por el que el $80 \%$ de la Generación X escoge un restaurante que aún no conoce, siguiendo la metodología 
presentada anteriormente se tienen las siguientes conclusiones.

La fuente más confiable para esta generación al momento de buscar información acerca del servicio de un restaurante que aún no conocen es la recomendación de terceras personas, ya que, casi el $85 \%$ de la Generación X afirma esto.

Para la Generación X, la importancia de leer los comentarios en redes sociales le es indiferente, además, no confía en los comentarios en redes sociales respecto a un restaurante que aún no conoce. Sin embargo, esta generación está de acuerdo en que confía en las recomendaciones de terceros respecto a lo mismo, esto claramente evidencia una preferencia hacia la publicidad boca - oído tradicional frente a la electrónica.

La Generación X también asegura que es más cómodo preguntar a algún conocido en lugar de revisar los comentarios en las redes sociales cuando quieren saber información de un restaurante que no conocen.

Finalmente, para el $92 \%$ de personas de la Generación X, las recomendaciones de terceros es el principal factor por el que escogen un restaurante que aún no conocen, mostrando favorecimiento total hacia este factor frente a los comentarios en redes sociales $u$ otros factores.

Basado en los datos expuestos, la hipótesis de que la publicidad boca - oído es el principal factor por el que el $80 \%$ de la Generación X escoge un restaurante que aún no conocen, se aprueba ya que 9 de cada 10 lo asegura.

Es claro que los comentarios en redes sociales para las personas de la Generación X, no es un factor a tener en cuenta, esto está respaldado con la baja confianza que tienen a las redes sociales, además de la baja importancia que le dan a los comentarios u opiniones. Asimismo, piensan que no es cómodo revisar los comentarios en redes antes de visitar un restaurante que no conocen.

En base a las conclusiones antes mencionadas, se recomienda motivar a los clientes de esta generación a recomendar el restaurante con sus conocidos, brindando beneficios para los clientes que visitan el restaurante habitualmente, apalancándose en los productos que son consumidos con un grado de constancia alto, por ejemplo, almuerzos. Funcionaría nombrar "embajadores" y ofrecer beneficios en los consumos habituales de estos, brindando cupones de descuento con códigos para sus conocidos, así se puede contabilizar cuántos clientes genera cada embajador.

\subsection{Características encontradas de los Millennials y de la Generación X en Cuenca - Ecuador}

El dispositivo preferido para los dos grupos de estudio es el mismo. El impacto que tiene el uso del celular es indiferente a la generación a la que pertenecen, además, el dispositivo con menor preferencia para la Generación X es la tableta, mientras que para los Millennials es la computadora.

En cuanto a las redes sociales, tanto Millennials como Generación X coinciden en que Facebook y YouTube son sus preferidas. En donde se encuentran diferencias es en el uso de Instagram y Twitter que son preferidas solo por los Millennials, mientras que Google+ es preferida solo por la Generación X.

Se encuentra diferencias en el número de redes que usan las dos generaciones, y similitudes en el tiempo y horario de preferencia. En promedio los Millennials (4 redes sociales) utilizan una red social más que la Generación X (3 redes sociales), sin embargo, el tiempo al día que le dedican al uso de redes es el mismo, de 1 a 2 horas diarias, coincidiendo también en las noches como el horario preferido para usarlas, de $18 \mathrm{~h} 00$ a $00 \mathrm{~h} 00$.

Al contrario de la Generación X, el uso de las redes sociales para los Millennials parece ir más allá de solo el contacto con sus amigos. Los dos grupos de estudio utilizan Facebook principalmente para chatear, además de usarlo cuando quieren buscar información acerca de restaurantes que no conocen. Por otro lado, se sabe que solo los Millennials siguen a las redes sociales de restaurantes locales provocando que sientan más confianza en restaurantes que tienen redes sociales frente a los que no las tienen; muy 
en contraste con la Generación X, que no usa redes sociales para seguir a restaurantes locales y le es indiferente si es que los mismos tienen redes sociales o no. Finalmente la percepción que se tiene de la publicidad en redes sociales es otro factor en el que los dos grupos coinciden, ya que a ambos les parece útil para conocer productos nuevos, sin embargo, para los Millennials esta publicidad sí resulta molesta.

Se recomienda utilizar la publicidad digital para informar acerca de los beneficios que ofrece el restaurante, en referencia al tipo de comida, los horarios de atención, si cuenta con menú para niños, menú para vegetarianos o veganos, etc., y evitar exhibir precios o descuentos en publicidad patrocinada.

Segmentar esta publicidad mediante el dispositivo de los usuarios, eligiendo el celular como medio de difusión, además de enfocarse en Facebook y YouTube. Potencializar el alcance de las publicaciones programadas durante la noche, desde las $18 \mathrm{H} 00$ hasta la media noche y mantener las redes sociales del restaurante actualizadas con toda la información que podrían demandar los clientes: dirección, ubicación, contacto, horario de atención, menú. 


\section{Referencias bibliográficas}

- Aguado Guadalupe, G., \& García García, A. (2009). Del Word-of-mouth al Mercadotecnia viral: aspectos claves de la comunicación a través de redes sociales. Revista Interdisciplinar de Ciencias de la Comunicación y Humanidades, 41-51.

- Arndt, J. A. (1967). Word of mouth advertising: a review of literature. Nueva York: Advertising Research Foundation. Recuperado el 5 de Octubre de 2017

- Burgueño, P. (2 de marzo de 2009). Pablo F. Burgueño. Recuperado el 28 de octubre de 2017, de https://www.pablofb.com/2009/03/clasificacion-de-redes-sociales/

- Caldevilla Domínguez, D. (03 de febrero de 2010). Las Redes Sociales. Tipología, uso y consumo de las redes 2.0 en la sociedad digital actual. Documentación de las Ciencias de la Información, 45-68. Recuperado el 10 de octubre de 2017, de Universidad Complutense Madrid: http://revistas.ucm.es/ index.php/DCIN/article/view/DCIN1010110045A

- Duan, Gu, \& Whinston. (2008). The dynamics of online word-of-mouth and product sales: and empirical investigation of the movie industry". En G. W. Duan. Recuperado el 7 de Octubre de 2017

- Goldsmith, \& Horowitz. (2006). Measuring motivations for online opinion seeking. Recuperado el 7 de Octubre de 2017

- Guevara, M. C., \& Morales, E. (2016). Repositorio Digital de la Universidad de Cuenca. Recuperado el 6 de julio de 2018, de http://dspace.ucuenca.edu.ec/bitstream/123456789/24474/1/Tesis.pdf

- Gutiérrez, A. (2016). MILLENNIALS EN LATINOAMÉRICA. Una perspectiva desde Ecuador. Barcelona: Editorial Ariel, S.A.

- Halstead, D. (2002). Negative word-of-mouth substitutive for or supplement to consumer complaints? Journal of Consumer Satisfaction, Dissatisfaction and Complaining Behavior., Vol. 15, p. 1-12. Recuperado el 28 de octubre de 2017

- Internacional, F. G. (Enero de 2017). Formacion Gerencial Internacional. Obtenido de http://blog. formaciongerencial.com/ranking-redes-sociales-sitios-web-aplicaciones-moviles-ecuador-2017/

- Islas, O. (noviembre de 2008). LA SOCIEDAD DE LA UBICUIDAD, LOS PROSUMIDORES Y UN MODELO DE COMUNICACIÓN PARA COMPRENDER LA COMPLEJIDAD DE LAS COMUNICACIONES DIGITALES. Red de Revistas Científicas de América Latina, el Caribe, España y Portugal. Recuperado el 13 de diciembre de 2017, de http://www.redalyc.org/ pdf/1995/199520724016.pdf

- Lau, G., \& Ng, S. (2001). Individual and situational factors influencing negative word-ofmouth behaviour. Canadian Journal of Administrative Sciences, Vol. 18, núm. 3., p. 163-178.

- Leon, S., \& Leslie, L. (2010). Riesgo Percibido. En S. Leon, \& L. Leslie, Comportamiento del consumidor (Décima ed., págs. 183-184). México: Pearson Educación. Recuperado el 11 de Octubre de 2017

- Moliner-Velázquez, B. (2012). Red de Revistas Científicas de América Latina, el Caribe, España y Portugal. Obtenido de Redalyc : http://www.redalyc.org/articulo.oa?id=43323186002

- Ovies, M. (28 de febrero de 2016). Medium. Obtenido de https://medium.com/@MarioOvies/eswhatsapp-una-red-social-8661 cbf8238a

- Rattinger, Á. (2013). NUEVO JUEGO, NUEVAS REGLAS. Mercadotecnia de vanguardia. México, D.F.: Ediciones Felou, S.A. Recuperado el 10 de noviembre de 2017

- Rubio, J. (19 de mayo de 2016). Verne. Recuperado el 25 de octubre de 2017, de https://verne.elpais. com/verne/2016/05/03/articulo/1462284105_813192.html 
- Sánchez, J. (05 de febrero de 2009). juanmercadotecnia.com. Obtenido de http://www. juanmercadotecnia.com/que-es-mercadotecnia-boca-oreja-oido/2009/02/05/

- Ureña, A., Ferrari, A., Blanco, D., \& Valdecasa, E. (diciembre de 2011). Observatorio da Sociedade da Información e a Modernización de Galicia. Recuperado el 3 de diciembre de 2017, de http://www. osimga.gal/export/sites/osimga/gl/documentos/d/20111201_ontsi_redes_sociais.pdf

- Velázquez Perea, L. E. (2016). Desarrollo de habilidades en el uso de las Tecnologías de la Información y la Comunicación. En L. E. Perea, Desarrollo de habilidades en el uso de las Tecnologías de la Información y la Comunicación. (pág. 30). México. Recuperado el 6 de Noviembre de 2017

- Villanueva, J., \& Armelini, G. (2007). El boca oreja electrónico: ¿Qué sabemos de esta poderosa herramienta de mercadotecnia? En J. Villanueva, \& G. Armelini, El boca oreja electrónico: ¿Qué sabemos de esta poderosa herramienta de mercadotecnia? (págs. 11-23). e-business Center PricewaterhouseCoopers \& IESE. Recuperado el 7 de Octubre de 2017, de http://www.ieseinsight. com/casos/Study_0092-S.pdf

- Webster, A. (2000). Estadística aplicada a los negocios y la economía. En A. Webster, Estadística aplicada a los negocios y la economía. (págs. 246-247). Bogotá: McGraw - Hill. Recuperado el 20 de Noviembre de 2017

\section{Anexos}

Anexo 1. Ficha técnica de investigación.

\begin{tabular}{|l|l|}
\hline \multicolumn{1}{|c|}{ Ficha técnica de investigación } \\
\hline Definición del problema. & $\begin{array}{l}\text { Desconocimiento de la importancia que se da a los comentarios en redes } \\
\text { sociales y a la publicidad boca-oído, como un factor de decisión en la } \\
\text { elección de restaurantes que aún no se conoce. }\end{array}$ \\
\hline Objetivo General. & $\begin{array}{l}\text { Conocer la importancia que se da a los comentarios en redes sociales y } \\
\text { a la publicidad boca oído, como un factor de decisión en la elección de } \\
\text { restaurantes que aún no se conoce. }\end{array}$ \\
\hline Objetivos Específicos. & $\begin{array}{l}\text { Conocer aspectos de importancia y el uso que dan a las redes sociales los } \\
\text { grupos de estudio. } \\
\text { Analizar la influencia que tiene la publicidad boca oído como un factor de } \\
\text { decisión en la elección de restaurantes que aún no conocen. } \\
\text { Estudiar la influencia que tienen los comentarios que realizan otros usuarios } \\
\text { en redes sociales como un factor de decisión en la elección de restaurantes } \\
\text { que aún no conocen. } \\
\text { Comparar la influencia que tienen los comentarios que dan otros usuarios } \\
\text { en redes sociales para visitar un restaurante que aún no se conoce frente a } \\
\text { la influencia de la publicidad boca oído para visitar un restaurante que aún } \\
\text { no se conoce. }\end{array}$ \\
\hline
\end{tabular}




\begin{tabular}{|l|l|}
\hline Grupo objetivo & $\begin{array}{l}\text { Millennials: Personas que viven en Cuenca-Ecuador, dentro del casco } \\
\text { urbano, de ambos géneros, con edades comprendidas entre } 25 \text { y } 35 \text { años, } \\
\text { con estudios universitarios. } \\
\text { Generación X: Personas que viven en Cuenca-Ecuador, dentro del casco } \\
\text { urbano, de ambos géneros, con edades comprendidas entre } 40 \text { y } 50 \text { años. }\end{array}$ \\
\hline Universo & $\begin{array}{l}\text { El universo es de } 142.575 \text { personas, correspondiente al } 28 \% \text { de la población } \\
\text { total del Cantón Cuenca. }\end{array}$ \\
\hline Diseño muestral & $\begin{array}{l}\text { Muestreo estratificado en el que los valores " } \mathrm{p} \text { " y "q" fueron tomados a } \\
\text { partir de una prueba piloto. }\end{array}$ \\
\hline Tamaño de la muestra & 295 personas. \\
\hline Técnica de recolección & Encuestas personales. \\
\hline Cobertura geográfica & Zonas urbanas del cantón Cuenca, Ecuador. \\
\hline Nivel de confianza & $95 \%$ \\
\hline Error máximo admitido & $5 \%$ \\
\hline
\end{tabular}

Elaboración: Los autores. 